\title{
Frame-Frame Matching for Realtime Consistent Visual Mapping
}

\author{
Kurt Konolige $^{1}$ and Motilal Agrawal ${ }^{1}$ \\ ${ }^{1}$ Artificial Intelligence Center, SRI International, 333 Ravenswood Ave., \\ 94025 Menlo Park, USA \\ \{konolige, agrawal\}@ai.sri.com \\ http://www.ai.sri.com/\{ konolige, agrawal
}

\begin{abstract}
Many successful indoor mapping techniques employ frame-to-frame matching of laser scans to produce detailed local maps, as well as closing large loops. In this paper, we propose a framework for applying the same techniques to visual imagery, matching visual frames with large numbers of point features. The relationship between frames is kept as a nonlinear measurement, and can be used to solve large loop closures quickly. Both monocular (bearing-only) and binocular vision can be used to generate matches. Other advantages of our system are that no special landmark initialization is required, and large loops can be solved very quickly.
\end{abstract}

\section{Introduction}

Visual motion registration is a key technology for many applications, since the sensors are inexpensive and provide high information bandwidth. In particular, we are interested in using it to construct maps and maintain precise position estimates for a mobile robot platform indoors and outdoors, in extended environments over loops of $>100 \mathrm{~m}$, and in the absence of global signals such as GPS - this is a classic SLAM (simultaneous localization and mapping) problem.

In a typical application, we gather images at frame rates, and extract hundreds of features in each frame for estimating frame to frame motion. Over the course of 100 $\mathrm{m}$, moving at $1 \mathrm{~m} / \mathrm{sec}$, we can have a thousand images and half a million features. The best estimate of the frame poses and feature positions is then a large nonlinear optimization problem. In previous research using laser rangefinders, one approach to this problem was to perform frame-to-frame matching of the laser scans, and keep only the constraints among the frames, rather than attempting to directly estimate the position of each scan reading (feature). This technique is used in the most successful methods for large-scale LRF map-making, FastSLAM [11][17] and Consistent Pose Estimation [8][12][15][16]. Using matching instead of feature estimation reduces the size of the nonlinear system by a large factor, since the features no longer enter into it.

In this paper, we present a frame-to-frame method for constructing maps from visual data. The main purpose of the paper is

- To show that precise realtime estimation of pose is possible, even in difficult outdoor environments, by visually matching frames that are spatially close (and not just temporally close, as in visual odometry).

- To show that a nonlinear frame-frame system is capable of quickly solving 
large-scale loop closure from visual information.

Precise estimation of frame pose is important in constructing good maps. In visual odometry, the pose is estimated by matching image features across several consecutive frames [1][18][19]. Current techniques achieve very precise results, but pose errors grow unbounded with time, even when the camera stays in the same area, because there is no matching of frames that are close in space, but not time. In contrast, the frame-frame matching techniques for LRF maps look for matches between frames that are spatially close, and obtain very precise floorplan results (see Figure 1). In a similar manner, our system computes the structure of spatially-coherent frame-frame visual constraints, and optimizes incrementally for realtime performance.

Recent research in vision-based SLAM has concentrated on solving the pose estimation problem for small areas by keeping track of feature positions. Davison's innovative technique [2] used a combined EKF over a small set of features. More recently, several approaches use a large number of features, each with its own independent EKF [4][23][21][22]. These methods rely on novel techniques for matching against a large database of features to achieve realtime performance. In both cases, the pose estimation accuracy suffers because of mismatches and imprecision in feature localization. We are investigating the relative performance of these techniques in small areas against our frame-frame matching, but do not yet have results to report here.

One advantage of the frame system is that no special initialization is required for landmarks, even in the monocular case, since we do not track the 3D position of landmarks. Instead, we use standard techniques in structure from motion to match image features and solve a projective system for the optimum local registration of frames and features [19][10][25]. Our novel technique is to derive a synthetic nonlinear measurement among frames alone that summarizes the registration. One obstacle to frame-frame constraints in the monocular case is that they are only partially con-

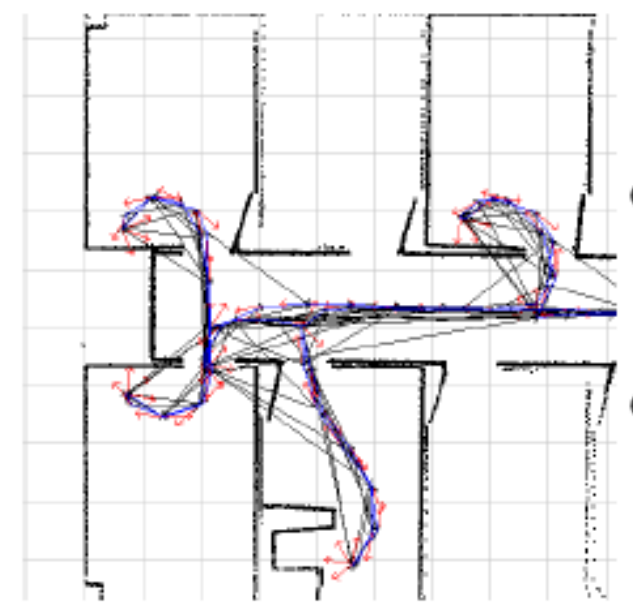

Figure 1 Frames linked by matching laser scans. Red arrows are frames, lines are links. Whenever there is a significant overlap of scans, a link is inserted, e.g., when the LRF sees through a doorway into the hall from separate rooms. 
strained (up to scale) - current laser scan systems, for example, cannot handle this case [13]. Our technique is more general, and can handle projective or even lessconstrained cases.

The frame-frame constraints are linked into a nonlinear system that mimics the much larger frame+landmark system. One of the weaknesses of current visual SLAM techniques with large numbers of landmarks is closing larger loops. In the course of a $100 \mathrm{~m}$ loop, there can be significant drift even with good frame-frame matching - in typical outdoor terrain our system has $2-4 \%$ error. After finding a loop-closing match, the frames in the loop can experience significant dislocation from their initial values. Our system computes the optimal nonlinear solution to the frame poses, in a fraction of a second, for large frame sets ( $>1 \mathrm{~K}$ frames).

Similar work in large-scale loop closure has recently emerged in undersea mapping using cameras [20][24] [26], although not in a realtime context. This research also uses frame-frame matching of images that are spatially close, but then filters the frame constraint system using a sparse information filter. In contrast, we construct an approximate nonlinear system, which can better conform to loop-closing constraints.

\section{Visual Matching and Nonlinear Systems}

Our approach derives from structure from motion theory of computer vision, in particular Sparse Bundle Adjustment (SBA). Of necessity we will present a short overview to introduce notation, and then apply it to frame-frame matching and the construction of the frame constraint system. Readers are urged to consult the excellent review in [25] for more detailed information.

\subsection{Sparse Bundle Adjustment}

We wish to estimate the optimal values of a set of parameters $\mathbf{x}$, given a set of measurements $\overline{\mathbf{z}}$. A measurement function $\mathbf{z}(\mathbf{x})$ describes the expected measurement from a given configuration of parameters $\mathbf{x}$. The error or cost induced by a given parameter set is

(1) $\varepsilon=\overline{\mathbf{z}}-\mathbf{z}(\mathbf{x})$.

If there are a set of independent measurements $\mathbf{z}_{i}$, each a Gaussian with covariance $W_{i}^{-1}$, then the MLE estimate $\hat{\mathbf{x}}$ minimizes the cost sum

(2) $E=\sum_{i} \varepsilon_{i}^{T} W_{i} \varepsilon_{i}$.

Since (2) is nonlinear, solving it involves reduction to a linear problem in the vicinity of an initial solution. At a value $\mathbf{x}, f$ can be approximated as

(3) $f(\mathbf{x}+\delta \mathbf{x}) \approx f(\mathbf{x})+g^{T} \delta \mathbf{x}+\frac{1}{2} \delta \mathbf{x}^{T} H \delta \mathbf{x}$,

where $g$ is the gradient and $H$ is the Hessian of $f$ with respect to $\mathbf{x}$. The minimum of $f$ is found by equating the derivative to zero. A further approximation gets rid of the second-derivative Hessian terms in favor of the Jacobian $J \equiv \partial \mathbf{z} / \partial \mathbf{x}$ (the GaussNewton normal equations):

(4) $J^{T} W J \delta \mathbf{x}=-J^{T} W \varepsilon_{0}$,

with $W$ the block-diagonal matrix formed from all the individual $W_{i}$. In the nonlinear 
case, one starts with an estimate $\mathbf{x}_{0}$, and iterates the linear solution until convergence to an estimate $\hat{\mathbf{x}}$. The Hessian has been approximated by

(5) $\quad H \approx J^{T} W J$.

It should be noted that $\hat{H}$ is also the inverse of the covariance of $\hat{\mathbf{x}}$, that is, the information matrix.

The general linear system (4) can be solved using a variety of methods, paying attention to step size to insure that there is a reduction in the total cost. In the application of (4) to camera frames and point features, SBA takes advantage of the sparse structure of $H$ to derive an efficient decomposition. Consider a set of camera frames $\mathbf{p}$ and features $\mathbf{q}$. The measurement functions $\mathbf{z}_{i j}\left(p_{i}, q_{j}\right)$ are the projection of features $q_{j}$ onto the frames $p_{i}$. Since only a small, bounded number of all features are seen by any camera frame, the pattern of the Jacobian $\partial \mathbf{z} / \partial \mathbf{x}$ is very sparse (the primary structure). If we reconstruct (4) by ordering the frames first and the features second, we get the following block structure:

(6) $\left[\begin{array}{ll}H_{p p} & H_{p q} \\ H_{q p} & H_{q q}\end{array}\right]\left[\begin{array}{l}\delta \mathbf{p} \\ \delta \mathbf{q}\end{array}\right]=\left[\begin{array}{l}-J_{p}^{T} W_{p} \varepsilon_{p} \\ -J_{q}^{T} W_{q} \varepsilon_{q}\end{array}\right]$,

where $H_{p p}$ and $H_{q q}$ are block-diagonal. Figure 2 shows a small example of three frames and two features.

Since the number of features is normally much larger than the number of frames, (6) would be easier to solve if it consisted just of the $H_{p p}$ section. In fact, it is possible to reduce (6) to the form

$$
\bar{H}_{p p} \delta \mathbf{p}=-\bar{g},
$$

where

$$
\begin{gathered}
\bar{H}_{p p} \equiv H_{p p}-H_{p q} H_{q q}^{-1} H_{q p} \\
\bar{g} \equiv J_{p}^{T} W_{p} \varepsilon_{p}-H_{p q} H_{q q}^{-1} J_{q}^{T} W_{q} \varepsilon_{q}
\end{gathered}
$$

After solving the reduced system (7) for the p's, the results can be back-propagated to find the q's, and the process iterated. Note that $\bar{H}_{p p}$ is the inverse covariance of the frame pose estimate.

It is important that in our application, the Hessian of the reduced system remains sparse as the number of frames grows (Figure 2, bottom). This is because each feature is seen by only a small, bounded number of frames, and so the number of elements of $\bar{H}_{p p}$ grows only linearly with the number of frames.

Another characteristic of the system (7) is that the choice of origin is arbitrary. All of the measurements are relative to a frame, and are preserved under arbitrary rigid transformations. So we can take any solution of (7) and transform it to a particular coordinate system, or equivalently, fix the pose of one of the frames. In fixing the pose, we eliminate the frame parameters, but keep it in the measurement equation as a fixed value for projection errors. 


\subsection{Frame-Frame Matching}

So far, the development has been a standard exposition of SBA. We will use (8) to calculate an incremental bundle adjustment [5] when adding a new camera frame to the system. For a large system, however, full SBA becomes computationally expensive, and more importantly, unstable when closing loops with significant offset. By using the idea of frame-frame matching from the LRF SLAM literature, we can convert a large nonlinear system of frame and feature measurements into a simpler (still nonlinear) system of frame-frame constraints. This conversion is only approximate, but we will show in a series of experiments that it produces good results.

Consider a simple system consisting of two frames $p_{0}$ and $p_{1}$, along with a large set of features $\mathbf{q}$ that are visible in both frames. Fix $p_{0}$ to be the origin, and calculate the estimated value $\hat{p}_{1}$ and its inverse covariance $\hat{H}_{11}$ (from (8)) using SBA. These two values summarize the complicated nonlinear relationship between $p_{0}$ and $p_{1}$ (constructed from the feature measurements) as a Gaussian PDF. This is exactly the PDF we would get from the measurement and its associated function

$$
\begin{gathered}
z\left(p_{1}\right)=p_{1} \\
\bar{z}=\hat{p}_{1},
\end{gathered}
$$

with inverse covariance $\hat{H}_{11}$ (see Appendix I). Here the measurement itself is $\hat{p}_{1}$, the estimated position of $p_{1}$. So we have compressed the effect of all variables $\mathbf{q}$ and their projections $\overline{\mathbf{z}}$ into a simple synthetic linear measurement on $p_{1}$.

Unfortunately (9) only holds when $p_{0}$ is the origin. What we would like is a measurement function that characterizes the relationship of $p_{0}$ and $p_{1}$ no matter where $p_{0}$ is located. The easiest way to do this is to measure the position of $p_{1}$ in

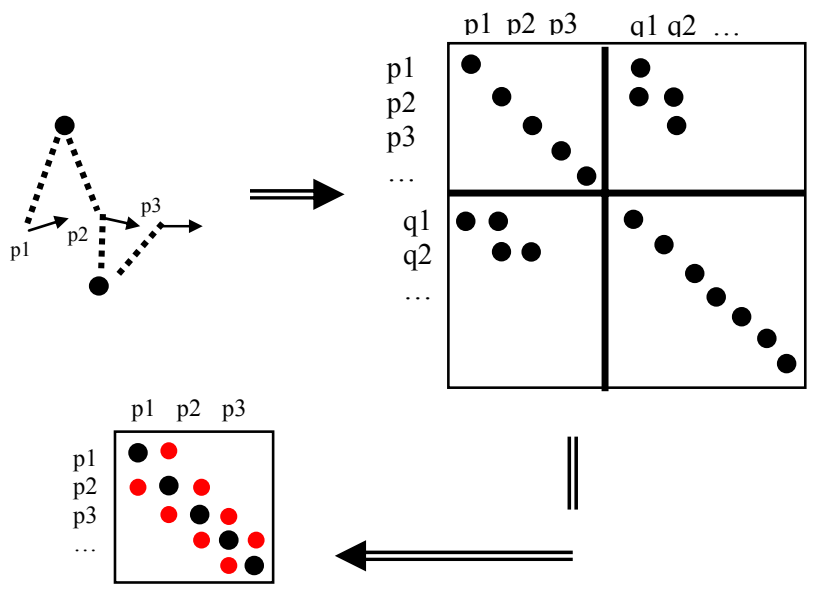

Figure 2 Top left: Three frames (arrows) and three features (dots). The dotted lines indicate that a feature is viewed from a frame. The Hessian is on the right, with the nonzero elements marked. Bottom left: reduced Hessian Hpp, showing banded structure. 
the frame $p_{0}$. Changing from the global frame to $p_{0}$ 's frame is accomplished by a homogenous transformation (see [3]); the value of $p_{1}$ in $p_{0}$ 's frame is denoted ${ }^{0} p_{1}$. Now the measurement and its function are

$$
\begin{gathered}
z\left(p_{0}, p_{1}\right)={ }^{0} p_{1} \\
\bar{z}={ }^{0} \hat{p}_{1}
\end{gathered}
$$

again with inverse covariance $\hat{H}_{11}$. This measurement function is no longer linear in the frame variables, but it is easy to see that when $p_{0}$ is the origin, it reduces to (9). More importantly, (10) produces exactly the same PDF for $p_{1}$ as does SBA, when both use the same (arbitrary) fixed value for $p_{0}$ (see Appendix I for a proof).

It is worth emphasizing the import of going from the large set of projective measurements $\mathbf{z}_{i j}\left(p_{i}, q_{j}\right)$ to the single measurement $z\left(p_{0}, p_{1}\right)={ }^{0} p_{1}$.

- The nonlinear system is reduced from several hundred variables (the features plus frames) to two variables (the frames).

- The nonlinear nature of the system is preserved, so that it is invariant to the absolute orientation and position of $p_{0}$ and $p_{1}$. This is in contrast to working with a reduced linear system (as in [20]), where re-linearization to correct bad initial angles is not possible.

- The measurement function (10) is a good approximation of the original system, as long as ${ }^{0} p_{1}$ is close to ${ }^{0} \hat{p}_{1}$.

- The measurement function (10) can be over-parameterized - the obvious case is for a monocular camera, in which the relation between $p_{0}$ and $p_{1}$ can be determined only up to a scale factor. The inverse covariance $\hat{H}_{11}$ has a null space and is not invertible, but is still useable in finding an ML estimate. This property is a great benefit, since we don't have to worry about finding a minimal representation, and can use frame-frame measurements even when they are only partially constrained, e.g, in the monocular case.

There is nothing that restricts frame-frame matching to working with just two frames - the reduction to pose differences works with any number of frames that have features in common. One frame must be chosen as the origin (say $p_{0}$ ); the general form is

$$
z(\mathbf{p})=\left[\begin{array}{c}
{ }^{0} p_{1} \\
0 \\
{ }^{0} p_{2} \\
\vdots \\
{ }^{0} p_{n}
\end{array}\right], \quad \overline{\mathbf{z}}=\left[\begin{array}{c}
0 \\
{ }^{0} \hat{p}_{1} \\
{ }^{0} \hat{p}_{2} \\
\vdots \\
{ }^{0} \hat{p}_{n}
\end{array}\right]
$$

with inverse covariance $\hat{H}_{p p}$. If all frames have at least one feature in common, then $\widehat{H}_{p p}$ has no nonzero elements, and the frames are fully connected. 


\section{Implementation}

The goal of this research is to implement a system that builds a map in realtime from visual information. The map system has the following elements:

1. Map state is the estimated pose $\hat{\mathbf{p}}$ of a set of frames that are extracted during camera motion.

2. Frame measurements are a set of measurements of the form (10) or (11).

3. Image features are point features extracted at each frame, associated with the frame. Image features do not enter into the map structure, and are kept only to perform image-to-image matching.

When a new frame is acquired, the system augments the map state $\hat{\mathbf{p}}$, and computes new frame measurements based on visual matching with other frames that are near in time and space. Then, a portion of the system is optimized using a subset of the frames and measurements. Where only local matches are made, a local area is optimized; for larger loops, the whole system may be involved. The optimization then updates the estimated pose $\hat{\mathbf{p}}$.

\subsection{Visual Matching}

The system expects calibrated cameras, and can use either monocular or binocular matching. For the binocular case, we match two frames; for monocular, three frames are used to preserve the relative scale. In either case, we use simple Harris points, and find putative matches by normalized cross-correlation of a small patch around the point. A robust RANSAC method [6] is used to find a good motion hypothesis. In the binocular case [18][1], three matched points are triangulated and then an absolute orientation step is used to estimate the $3 D$ motion. The estimate is scored by projecting all features back onto the images and counting the number of inliers.

For monocular motion, the 5-point method of [18] is used to hypothesize an essential matrix for the first and third frames, and the second frame is estimated from a three-point resection [9]. Again projection is used to find the maximum number of inliers.

For the best hypothesis, the SBA method of Section 2.1 optimizes the whole system, and at the same time computes the Hessian for the frame-frame constraint (10) or (11). Note that in the monocular case, the Hessian has a null space of dimension one, since the overall scale is indeterminate. This exactly characterizes the relative placement of the three frames, while leaving open the scale.

\subsection{Data Association}

Visual matching takes place independent of the state of the map system, producing frame-frame measurements. One of the critical system choices is deciding which measurements to add when a new frame is added. For this paper we adopted a simple scheme that is efficient and produces reasonable results. First, we add a set of measurements that connect to the previous $N$ frames, where $N$ is a small number, typically 1 to 5 . Then, we add at most one measurement to any close, non-recent frame. These additions keep the map estimate consistent locally. Finally, we search for longerrange measurements that close larger loops, and add one of these if appropriate. 
For short-range motion, spatially nearby frames can be identified if they are close in the graph of measurements [8]. For longer-range loops, we are investigating the use of more invariant features to reliably identify closure hypotheses, e.g., the method of [24].

\subsection{Computation}

The more demanding case is binocular, because features must be extracted from two images, and matched across the images as well as with previous images. Figure 3 presents a breakdown of the computation on a $2 \mathrm{GHz}$ Core Duo Pentium M, using $512 \times 384$ images and approximately 500 points in each image. For each new frame, the first three computations must be performed to connect to the previous frame. For more matches to previous frames, only the motion estimation step needs to be done; for matches to close frames, both feature tracking and motion estimation are needed. The system can perform several visual matches within a $15 \mathrm{~Hz}$ cycle, with visual matching partitioned between the two cores. Updating the map system takes very little time compared to the matching stage: Figure 3 also shows some timings for medium to large systems. The system is implemented and runs on an outdoor robot that uses stereo to autonomously build maps in off-road environments [14].

\section{Results}

We performed two sets of experiments, one with simulated data where the ground truth is known, and one with a dataset from an outdoor robot moving about $100 \mathrm{~m}$ in a loop.

\subsection{Simulated monocular system}

In this experiment we compare the frame-frame system to the standard SBA method on a local loop, to test its accuracy. The motion is circular, with the camera looking in the direction of motion. For the frame system, we use 3-frame constraints to propagate relative scale. We varied the density of constraints for each frame, from 1 to 5 . Figure 4 shows typical results, with the SBA motion in blue, and the frame system in red. For the frame system, the last few frames were matched against the first few to create loop constraints. For SBA, image feature tracks average 7 frames,

\begin{tabular}{|l|c|}
\hline Algorithm & CPU time \\
\hline \hline Feature extraction and stereo matching & $25 \mathrm{~ms}$ \\
\hline Visual matching & $24 \mathrm{~ms}$ \\
\hline Motion estimation (per constraint) & $16 \mathrm{~ms}$ \\
\hline System optimization & \\
\hline 80 frames & $30 \mathrm{~ms}$ \\
\hline 330 frames & $100 \mathrm{~ms}$ \\
\hline 660 frames & $220 \mathrm{~ms}$ \\
\hline 1330 frames & $340 \mathrm{~ms}$ \\
\hline
\end{tabular}

Figure 3 Computation times for the main parts of the mapping system. $2 \mathrm{GHz}$ Pentium M, 512x384 images, $\sim 500$ points per image. 


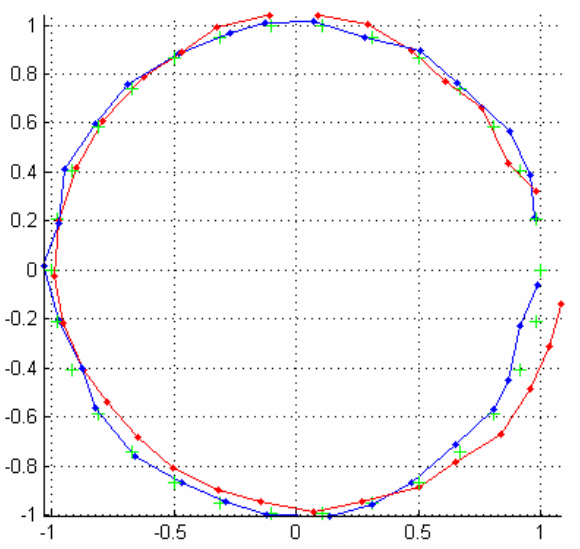

Figure 4 Typical circular motion estimate at high noise levels, projected onto the $\mathrm{XY}$ plane. Green crosses are the ground truth frame positions. Blue is full SBA, red is the frame system with 2 links ( 3 pixels image error).

and no loop-closure matching is used. Note the accuracy of the frame system, even though it uses several orders of magnitude fewer measurements.

Figure 5 shows statistics for varying amounts of Gaussian noise on the image points. The error is measured as the rms distance of poses from their ground-truth positions, averaged over 20 runs. Since the scale and placement of the result is not constrained, we did a final minimization step, using a rigid transformation and scale to bring it into correspondence with ground truth.

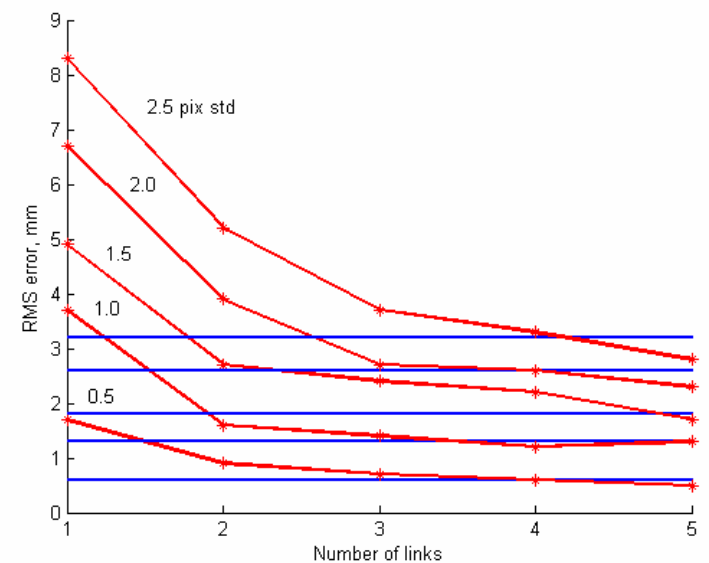

Figure 5 RMS error in pose $(\mathrm{mm})$ for circular motion, for different numbers of links and image noise. Red lines are frame system, blue lines are full SBA. Bottommost red and blue lines are for 0.5 pixels image noise, topmost are for 2.5 pixels noise. 
The key aspect of Figure 5 is that the reduced system results are as good as or better than SBA, especially at 4 and 5 links. As the image noise increases, SBA does increasingly worse because it is open-ended, while the frame system degrades less. Note that these results are much more accurate than the reduced system in [7], which uses an averaging technique between adjacent frames, and neglects longer links. This experiment validates the use of the frame system for high-accuracy motion estimation in a local area.

\subsection{Outdoor stereo system}

We conducted a large outdoor experiment, using a mobile robot with a fixed stereo pair, inertial system, and GPS (Figure 6). The FOV of each camera was about $100^{\circ}$, and the baseline was $12 \mathrm{~cm}$; the height above ground was about $0.5 \mathrm{~m}$, and the cameras pointed forward at a slight angle. This arrangement presents a challenging situation: wide FOV and short baseline make distance errors large, and a small offset from the ground plane makes it difficult to track points over longer distances.

The test course covered about $110 \mathrm{~m}$, and concluded at the spot where it started. Figure 8 shows the global position, as determined by GPS and inertial systems, and the poses computed from the frame system - 1172 frames with average spacing of just under $0.1 \mathrm{~m}$. The run started from the origin, went across and diagonally down to the lower right, then came back below the original track. The frame system did a reasonable job, with an accumulated error of about $3 \mathrm{~m}$ over the run. The angle gets off a bit on the return trip, and consequently the loop meeting point is overrun. Note that the travel distance was very close $-110.9 \mathrm{~m}$ for GPS, 110.02 for VO.

To correct the accumulated error, we closed the loop by matching the last frame to the first. Our visual matching algorithm was used to find the constraint, since the two frames were close. The visual results now track GPS much more closely (Figure 8), and in fact are better than GPS right around the origin, where GPS is off by almost 1 $\mathrm{m}$. The path length has not changed, but the angular error along the path has been

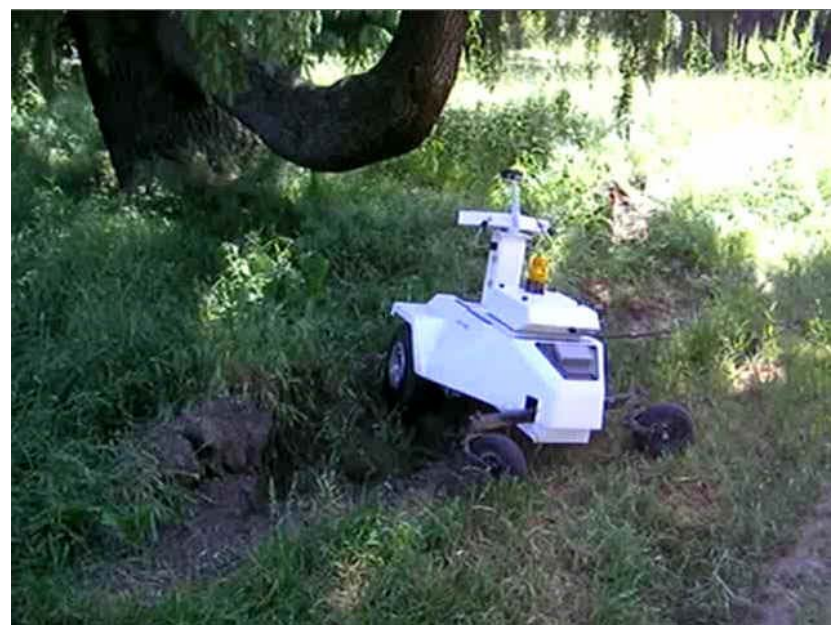

Figure 6 Outdoor robot in typical terrain. Robot is part of a DARPA project, Learning Applied to Ground Robotics. Two stereo systems are on the upper crossbar. 

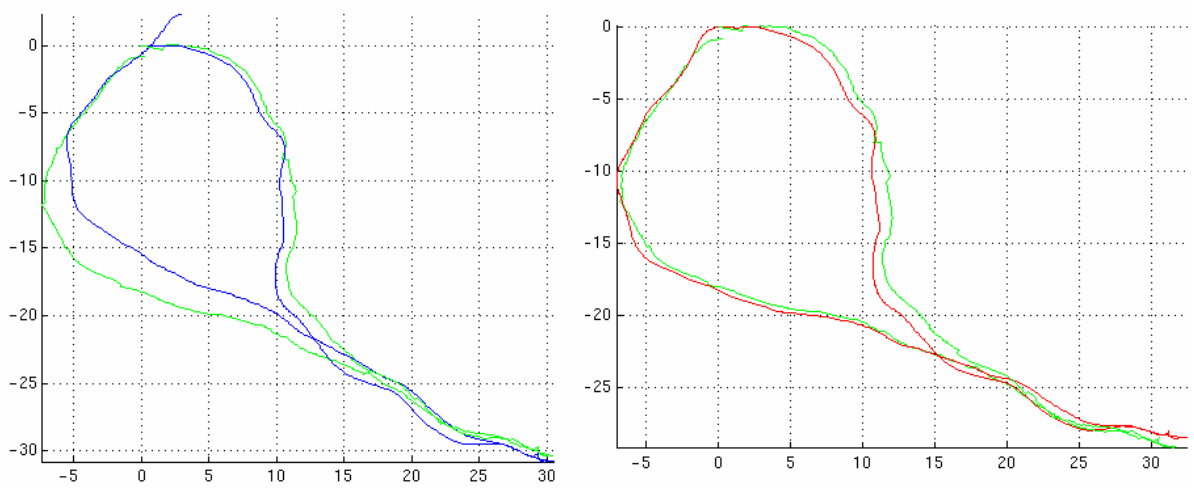

Figure 8 Top: Frame system of an extended outdoor run. Global pose from GPS and inertial sensors (green), frame system in blue. Note the overshoot at the end of the loop. Bottom: Frame system with loop closure, in red.

corrected and spread evenly, based on the loop closure.

Even more interesting is the data from the $\mathrm{Z}$ (earth-normal) direction, in Figure 7. The GPS/inertial data drifts considerably over the run, ending at almost $-7 \mathrm{~m}$. The frame data (blue) also drifts, but to much less extent, ending at $-3 \mathrm{~m}$. Adding loop closure corrects the drift at the origin, and pulls up the rest of the path as well.

Given our timing results, it is possible to perform loop closure online. But we can reduce the computational load still further by reducing the number of frame-frame constraints. To do this, we use the same technique as in (6)-(8), but add to the features $\mathbf{q}$ all the frames between two endframes. The reduced system (7) then contains just the two endframes, and we construct a synthetic measurement between these two, as in (9). For this experiment, two reductions were chosen, based on $1 \mathrm{~m}$ and $4 \mathrm{~m}$ distance. Starting with the first frame, we find the next frame that is greater than this distance or more than $10^{\circ}$ different in angle. We then reduce all frames in between, add the loop closure constraint, and solve the system. The reduction leads to systems of 126 and 41 poses, respectively. The results are shown in Figure 9. The blue crosses, for the 126-frame system, recover almost exactly the form of the original frame system. Even with a 10-fold reduction in the number of frames, from 1172 to 126 , the system produces excellent results. With only 41 poses, errors start to appear at a small scale, although the overall shape still remains very good.

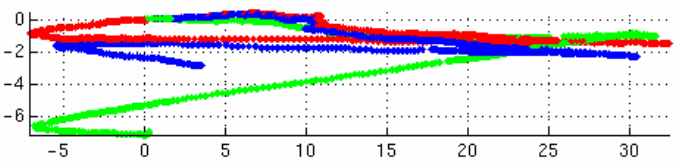

Figure 7 Comparison of $\mathrm{Z}$ vs. $\mathrm{X}$ motion of GPS (green), frame system (blue), and frame system with loop closure (red). 


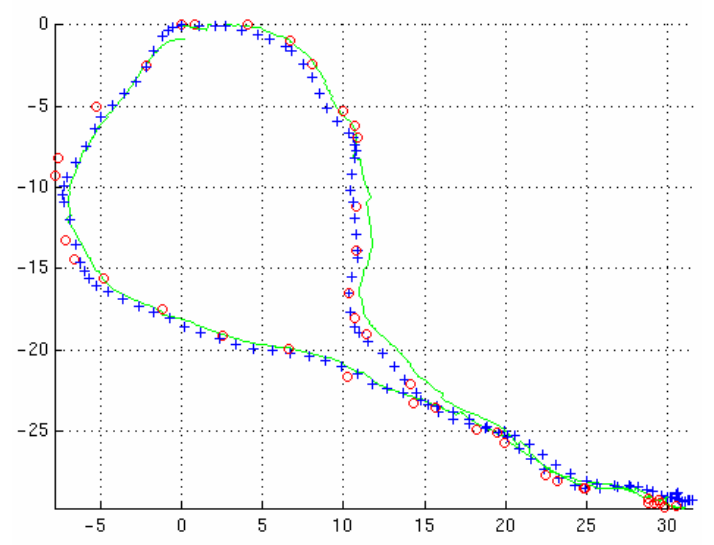

Figure 9 Loop-closing with reduced number of poses. The blue crosses are for $1 \mathrm{~m}$ distance between frames (126 poses), the red circles for $4 \mathrm{~m}$ (41 poses). Green is global GPS pose.

\section{Discussion}

This paper lays the foundation for an online method of consistent motion estimation, one that takes into account global constraints such as loop closure, while preserving the fine structure of motion. It is based on proven methods from the laser scan-matching SLAM literature, adapted using structure-from-motion techniques. A careful analysis of the structure of measurements in SBA shows how to construct new, nonlinear frame-frame constraints in a theoretically motivated way. The resultant systems can be almost as accurate as the original system, while enjoying large speedups in computation.

One of the nice properties of the frame-frame system is that it keeps the set of camera frames, so that reconstruction (e.g., dense stereo) can be performed. This is in contrast to EKF methods [1][4][23][21][22], which keep only a current estimate of the camera pose.

While we show that online consistent estimation is possible, we have not yet developed a full system that exploits it. Such a system would have a map management component, for keeping track of images associated with poses, and deciding when to match the current image against others for loop closure. It would also need more robust features for wide-baseline matching. It is our goal to construct a complete system that performs online map-making over large areas, using just visual input.

Our current system uses the robust VO component to keep track of position in varied outdoor terrain, including under tree cover where GPS does not work very well. Our system performed the best in a final evaluation of the DARPA Learning Applied to Ground Robotics project in June of 2006, using VO to keep track of its position over a challenging course. 


\section{Appendix I}

Let $x_{0}, x_{1}$ and $\mathbf{q}$ be a set of variables with measurement equation $z\left(x_{0}, x_{1}, \mathbf{q}\right)$ and measurement $\bar{z}$ and cost function

(I1) $\sum \Delta z_{i}^{T} W_{i} \Delta z_{i}$.

For $x_{0}$ fixed at the origin, let $\hat{H}_{11}$ be the Hessian of the reduced form of I1, according to (8). We want to show that the cost function

(I2) $\sum \Delta z^{\prime T} \hat{H}_{11} \Delta z^{\prime}$

has approximately the same value at the ML estimate $x_{1}^{*}$, where $z^{\prime}\left(x_{0}, x_{1}\right)={ }^{0} x_{1}$ and $\bar{z}^{\prime}=x_{1}^{*}$. To do this, we show that the likelihood distributions are approximately the same.

The cost function (I1) has the joint normal distribution

(I3) $P(\hat{\mathbf{z}} \mid \mathbf{x}) \propto \exp \left(-\frac{1}{2} \sum \Delta z_{i}^{T} W_{i} \Delta z_{i}\right)$.

We want to find the distribution (and covariance) for the variable $x_{1}$. With the approximation of $f(\mathbf{x}+\delta \mathbf{x})$ given in (3), convert the sum of (I3) into matrix form.

$$
\begin{aligned}
&(\hat{\mathbf{z}}-f(x, \mathbf{q}))^{T} W(\hat{\mathbf{z}}-f(x, \mathbf{q})) \\
&(\mathrm{I} 4) \quad \approx\left(\hat{\mathbf{z}}-f\left(x^{*}, \mathbf{q}^{*}\right)-J \delta\right)^{T} W\left(\hat{\mathbf{z}}-f\left(x^{*}, \mathbf{q}^{*}\right)-J \delta\right) \\
&= \delta x^{T} \hat{H}_{11} \delta x-2 \Delta z^{*} W J \delta+\text { const }
\end{aligned}
$$

where we have used the result of (7) and (8) on the first term in the last line. As $\Delta z^{*}$ vanishes at $x^{*}, \mathbf{q}^{*}$, the last form is quadratic in $x$, and so is a joint normal distribution over $x$. From inspection, the covariance is $\hat{H}_{11}^{-1}$. Hence the ML distribution is

(I5) $P(x \mid \hat{\mathbf{z}}) \propto \exp \left(-\frac{1}{2}\left(x-x^{*}\right)^{T} \hat{H}_{11}\left(x-x^{*}\right)\right)$.

The cost function for this PDF is (I2) for $x_{0}$ fixed at the origin, as required.

When $x_{0}$ is not the origin, the cost function (I1) can be converted to an equivalent function by transforming all variables to $x_{0}$ 's coordinate system. The value stays the same because the measurements are localized to the positions of $x_{0}$ and $x_{1}$ - any global measurement, for example a GPS reading, would block the equivalence.

Thus, for arbitrary $x_{0}$, (I5) and (I3) are approximately equal just when $x_{1}$ is given in $x_{0}$ 's coordinate system. This is the exact result of the measurement function $z^{\prime}\left(x_{0}, x_{1}\right)={ }^{0} x_{1}$.

\section{References}

[1] Agrawal, M. and Konolige, K. Real-time Localization in Outdoor Environments using Stereo Vision and Inexpensive GPS. Proceedings of the International Conference on Pattern Recognition (2006)

[2] Davison, A. Realtime SLAM with a single camera. Proc. ICCV, 2003. 
[3] Craig, J.J. Introduction to robotics: mechanics and control, AddisonWesley, MA, 1989.

[4] Eade, E. and T. Drummond, "Scalable Monocular SLAM," CVPR (2006).

[5] Engels, C., H. Stewénius, D. Nistér. Bundle Adjustment Rules. Photogrammetric Computer Vision (PCV), September 2006.

[6] Fischler, M. and R. Bolles. Random sample consensus: a paradigm for model fitting with application to image analysis and automated cartography. Commun. ACM., 24:381-395, 1981.

[7] Govindu, V. M. Lie-algebraic averaging for globally consistent motion estimation. In Proc. IEEE Conference on Computer Vision and Pattern Recognition, June 2004.

[8] Gutmann, J. S. and K. Konolige. Incremental Mapping of Large Cyclic Environments. In CIRA 99, Monterey, California, 1999.

[9] Haralick, R., C. Lee, K. Ottenberg, M. Nolle. Review and analysis of solutions of the three point perspective pose estimation problem. IJCV (1994).

[10] Hartley, R. and A. Zisserman. Multiple View Geometry in Computer Vision, Second Edition. Cambridge University Press, 2003.

[11] Hahnel, D., W. Burgard, D. Fox, and S. Thrun. An efficient FastSLAM algorithm for generating maps of large-scale cyclic environments from raw laser range measurements. In Proc. of the IEEE/RSJ Int. Conf. on Intelligent Robots and Systems (IROS), 2003.

[12] Konolige, K. Large-Scale Map Making. In Proc. AAAI, San Jose, California (2004).

[13] Konolige, K. SLAM via variable reduction from constraint maps. In Proc. ICRA, March 2005.

[14] Konolige, K., Agrawal, M., Bolles, R., Cowan, C., Fischler, M. and Gerkey, B. Outdoor Mapping and Navigation using Stereo Vision. Proceedings of the International Symposium on Experimental Robotics, Brazil (2006)

[15] Lu, F. and E. E. Milios. Globally consistent range scan alignment for environment mapping. Autonomous Robots, 4(4), 1997.

[16] Montemerlo, M. and S. Thrun. Large-scale robotic 3-d mapping of urban structures. In ISER, Singapore, 2004.

[17] Montemerlo, M., Thrun, S., Koller, D. and Wegbreit, B. FastSLAM: A factored solution to the simultaneous localization and mapping problem. AAAI (2002).

[18] Nister, D., O. Naroditsky, and J. Bergen. Visual odometry. In Proc CVPR, June 2004.

[19] Olson, C. F., L. H. Matthies, M. Schoppers, and M. W. Maimone. Robust stereo egomotion for long distance navigation. In Proc CVPR, 2000.

[20] Ryan, E., Singh, H., and J. Leonard, "Exactly sparse delayed state filters," in Proc ICRA, April 2005.

[21] Se, S., Lowe, D. G., and J. J. Little. Vision-Based Global Localization and Mapping for Mobile Robots. IEEE Transactions on Robotics, 21(3), June 2005.

[22] Se, S., Lowe, D. G., and J. J. Little. Mobile robot localization and mapping with uncertainty using scale-invariant visual landmarks, Int. J. Robot. Res.21(8), Aug. 2002.

[23] Sim, R., P. Elinas, M. Griffin, and J. J. Little. Vision-based slam using the raoblackwellised particle filter. In IJCAI Workshop on Reasoningwith Uncertainty in Robotics, 2005.

[24] Singh, H., C. Roman, O. Pizarro, and R. Eustice, Advances in high-resolution imaging from underwater vehicles, Intl. Symp. on Robotics Research, October 2005

[25] Triggs, B., P. McLauchlan, R. Hartley, and A. Fitzgibbon. Bundle adjustment - a modern synthesis. In Vision Algorithms: Theory \& Practice. Springer-Verlag, 2000.

[26] Walter, M, Ryan, E, and J. J. Leonard. A provably consistent method for imposing exact sparsity in feature-based SLAM information filters. In Proc. ISRR, October 2005. 